\title{
Damping Rate of a Hard Photon in a Relativistic Plasma
}

\author{
M.H. Thoma \\ Institut für Theoretische Physik, Universität Giessen, \\ 35392 Giessen, Germany
}

(September 15, 2021)

\begin{abstract}
The damping rate of a hard photon in a hot relativistic QED and QCD plasma is calculated using the resummation technique by Braaten and Pisarski.

PACS numbers: $12.38 . \mathrm{Mh}, 12.38 . \mathrm{Bx}$
\end{abstract}

\section{INTRODUCTION}

Damping rates of hard particles in a relativistic plasma at high temperature attracted much interest recently [1] 19 . For instance, the damping rate of a quark in a QCD plasma is related to important quantities of the quark-gluon plasma, such as mean free paths, thermalization times [18,20], viscosity [18,21, and stopping power [5, 7, 22 25]. Damping rates also represent simple tests for methods of the finite temperature field theory, probing e.g. the infrared behavior [16] and gauge dependence [10,11, 13, 14,26 28] of the methods.

Hard quarks and gluons, i.e. partons with energies $E \gtrsim T$, as well as hard electrons and muons in a relativistic QED or QCD plasma are damped to lowest order by elastic scattering off the thermal particles mediated by the exchange of a gauge boson. Owing to the long range interaction (massless gauge boson) those processes exhibit a quadratic infrared singularity. The infrared behavior can be improved by resumming the gauge boson self energy in the "Hard Thermal Loop" approximation, leading to an effective gauge boson propagator containing screening effects (Debye screening). This prescription is a special case of the Braaten-Pisarski resummation technique 29] which produces consistent results, i.e. gauge independent results for observables that are complete to leading order in the coupling constant. The use of the effective gauge boson propagator, containing the square of the coupling constant $g^{2}$ in the denominator, reduces the order of the damping rates, naively expected to be proportional to $g^{4}$, to $g^{2}$, which was called anomalous (large) damping 2 24]. At the same time the quadratic infrared singularity is replaced by a logarithmic one, reflecting the absence of static magnetic screening in the effective gauge boson propagator. (This result led to speculations that these rates might become finite by higher order effects such as a non-perturbative magnetic screening mass or self consistent damping [16] or that these rates cannot be regarded as direct observables but should be modified by transport factors [2], 18, .)

Here we consider the damping of a high energy photon with four momentum $P=\left(p_{0}, \mathbf{p}\right)$ and $p=|\mathbf{p}| \gg T$ in a relativistic plasma of electrons and positrons or quarks at a temperature much higher than the bare masses of those particles. In contrast to partons or leptons the leading order damping mechanism is not caused by elastic scattering since photons show no self interaction. To lowest order the photons are damped by Compton scattering and pair creation processes shown in Fig.1. Neglecting the fermion masses the corresponding rate suffers from a logarithmic infrared singularity. However, this divergence is screened by medium effects again leading to an effective temperature dependent fermion mass of the order of $g T$ which cuts off the singularity much more effectively than the bare mass. Since the divergence is only logarithmic utilizing a bare propagator it disappears completely taking the effective one into account, analogously to the energy loss of an energetic parton in the quark-gluon plasma [7,22, 24. Thus the introduction of a magnetic screening mass, self consistently damped fermion propagators, or transport factors is unnecessary in the case of photon damping.

Similarly to the energy loss the photon damping rate can be derived consistently by applying the Braaten-Yuan prescription [30]. For this purpose we decompose the rate into a soft contribution, which requires an effective fermion propagator, and a hard contribution, where bare propagators are sufficient. The decomposition is accomplished by introducing a separation scale $\Lambda$ in the momentum of the exchanged fermion which drops out at the end after adding soft and hard contributions if $\Lambda$ is restricted by $g T \ll \Lambda \ll T$, which is possible in the weak coupling limit $g \ll 1$. (In the case of partons or leptons the hard contribution can be neglected since the corresponding damping rates are dominated by small momentum transfers.)

The damping rate is defined as the imaginary part of the photon dispersion relation, $\gamma=-\operatorname{Im} E(p)$, where the dispersion relation for a real, i.e. transverse, photon follows from

$$
p_{0}^{2}-p^{2}-\Pi_{T}\left(p_{0}, p\right)=0
$$


where the transverse part of the photon self energy is given by

$$
\Pi_{T}\left(p_{0}, p\right)=\frac{1}{2}\left(\delta_{i j}-\frac{p_{i} p_{j}}{p^{2}}\right) \Pi_{i j}\left(p_{0}, p\right) .
$$

In the case of no overdamping $(p \ll \gamma)$ we get from (11)

$$
\gamma=-\frac{1}{2 p} \operatorname{Im} \Pi_{T}\left(p_{0}=p, p\right)
$$

The damping rate is related to the decay rate $\Gamma_{d}$ by $\gamma=\Gamma_{d} / 2$, where $\Gamma_{d}$ follows from a momentum integration over matrix elements and distribution functions (see (8) and (9)) [31]. We will calculate the soft contribution contribution starting from (3), whereas the hard contribution starting from the decay rate.

The soft contribution $\left(k_{0}, k<\Lambda\right)$ follows from the photon self energy shown in Fig.2, where the blob denotes the effective fermion propagator containing the one-loop fermion self energy in the Hard Thermal Loop approximation [29]. (According to the rules of the Braaten-Pisarski method we do not need an effective vertex or two effective fermion propagators at the same time since the external photon is hard.) Cutting through the blob and a bare propagator we see that these diagrams correspond to Compton scattering and pair creation via the exchange of a dressed (collective) fermion.

First we will consider the damping of a photon in a hot relativistic QED plasma. The photon self energy shown in Fig.2 is given by

$$
\Pi_{\mu \nu}=2 i e^{2} \int \frac{d^{4} K}{(2 \pi)^{4}} \operatorname{tr}\left[\gamma_{\mu} S(Q) \gamma_{\nu} S^{\star}(K)\right],
$$

where $S^{\star}(K)$ indicates the effective fermion propagator, the factor of 2 comes from adding the both diagrams in Fig.2, and $Q=P-K$. It is convenient to use the helicity representation of the fermion propagator given in [32, 33]. (The effective electron propagator differs from the quark propagator only by the effective mass $m_{e}^{2}=e^{2} T^{2} / 8$ compared to $m_{q}=g^{2} T^{2} / 6$.)

Evaluating the trace over the gamma matrices we get for the transverse part of the polarization tensor

$$
\Pi_{T}=2 i e^{2} \int \frac{d^{4} K}{(2 \pi)^{4}}\left[\frac{1}{D_{+}(K)}\left(\frac{1-V}{d_{+}(Q)}+\frac{1+V}{d_{-}(Q)}\right)+\frac{1}{D_{-}(K)}\left(\frac{1+V}{d_{+}(Q)}+\frac{1-V}{d_{-}(Q)}\right)\right],
$$

where the functions $D_{ \pm}(K)$ and $d_{ \pm}(Q)$ are given for example by Kapusta et al. [33] and $V=(\mathbf{p} \cdot \mathbf{k})(\mathbf{p} \cdot \mathbf{q}) /\left(p^{2} q k\right)$.

Using the imaginary time formalism the integration over $k_{0}$ in (5) is replaced by a discrete sum, which can be evaluated most easily by adopting the spectral representation for the fermion propagators [32, 33]. Using furthermore $p \gg T \gg k_{0}, k$ we find

$$
\operatorname{Im} \Pi_{T}\left(p_{0}, p\right)=-\frac{e^{2}}{4 \pi} \int_{0}^{\Lambda} d k \int_{-k}^{k} d \omega\left[(k-\omega) \beta_{+}(\omega, k)+(k+\omega) \beta_{-}(\omega, k)\right],
$$

where $\beta_{ \pm}=-\operatorname{Im} D_{ \pm}^{-1} / \pi$ are the discontinuous parts of the spectral functions belonging to the effective fermion propagator. This expression agrees up to some prefactors with the photon production rate. Thus following Kapusta et al. [33] we end up with

$$
\gamma_{s o f t}=\frac{\pi}{4} \frac{\alpha^{2} T^{2}}{p} \ln \frac{\Lambda^{2}}{\pi \alpha T^{2}}
$$

The hard contribution, for which the momentum of the exchanged fermion is larger than $\Lambda$, can be calculated most conveniently from the matrix elements according to Fig.1 of Compton scattering

$$
\begin{gathered}
\gamma_{\text {hard }}^{\text {comp }}=\frac{1}{4 p} \int \frac{d^{3} k}{(2 \pi)^{3} 2 k} n_{F}(k) \int \frac{d^{3} p^{\prime}}{(2 \pi)^{3} 2 p^{\prime}}\left[1+n_{B}\left(p^{\prime}\right)\right] \int \frac{d^{3} k^{\prime}}{(2 \pi)^{3} 2 k^{\prime}}\left[1-n_{F}\left(k^{\prime}\right)\right] \\
(2 \pi)^{4} \delta^{4}\left(P+K-P^{\prime}-K^{\prime}\right) 4\left\langle|\mathcal{M}|^{2}\right\rangle_{\text {comp }}
\end{gathered}
$$

and pair creation

$$
\begin{gathered}
\gamma_{\text {hard }}^{\text {pair }}=\frac{1}{4 p} \int \frac{d^{3} k}{(2 \pi)^{3} 2 k} n_{B}(k) \int \frac{d^{3} p^{\prime}}{(2 \pi)^{3} 2 p^{\prime}}\left[1-n_{F}\left(p^{\prime}\right)\right] \int \frac{d^{3} k^{\prime}}{(2 \pi)^{3} 2 k^{\prime}}\left[1-n_{F}\left(k^{\prime}\right)\right] \\
(2 \pi)^{4} \delta^{4}\left(P+K-P^{\prime}-K^{\prime}\right) 2\left\langle|\mathcal{M}|^{2}\right\rangle_{\text {pair }} .
\end{gathered}
$$


Here momenta with a prime belong to outgoing particles. The factors in front of the amplitudes, averaged over initial states and summed over final ones, come from summing over the possible states of the incoming thermal particle. The matrix elements using Mandelstam variables, $s=(P+K)^{2}, t=\left(P-P^{\prime}\right)^{2}, u=-s-t$, are given by [34]

$$
\begin{aligned}
\left\langle|\mathcal{M}|^{2}\right\rangle_{\text {comp }} & =-2 e^{4}\left(\frac{u}{s}+\frac{s}{u}\right), \\
\left\langle|\mathcal{M}|^{2}\right\rangle_{\text {pair }} & =2 e^{4}\left(\frac{u}{t}+\frac{t}{u}\right) .
\end{aligned}
$$

In order to evaluate the integrations over the final states in (8) and (9) we assume $p^{\prime} \gg T$ and $k^{\prime} \gg T$, which is justified since $p>p^{\prime}+k^{\prime} \gg T$ and the phase space for $p^{\prime} \stackrel{\sim}{\sim}$ or $k^{\prime} \lesssim T$ is unfavorable [33]. Thus we may assume $1+n_{B}\left(p^{\prime}\right) \simeq 1-n_{F}\left(p^{\prime}\right) \simeq 1-n_{F}\left(k^{\prime}\right) \simeq 1$ corresponding to the Boltzmann approximation for the distribution functions, e.g. $1+n_{B}\left(p^{\prime}\right)=\exp \left(p^{\prime} / T\right) n_{B}\left(p^{\prime}\right) \simeq 1$, also used by Kapusta et al. [33] in the case of photon production, where, however, an integration over the initial states was considered. This approximation simplifies the expressions (8) and (9) considerably because we can now evaluate the integrals over $p^{\prime}$ and $k^{\prime}$ transforming to the center of mass system using the Lorentz invariant phase space factor [34]

$$
d Q=(2 \pi)^{4} \delta^{4}\left(P+K-P^{\prime}-K^{\prime}\right) \frac{d^{3} p^{\prime}}{(2 \pi)^{3} 2 p^{\prime}} \frac{d^{3} k^{\prime}}{(2 \pi)^{3} 2 k^{\prime}}=\frac{d t}{8 \pi s} .
$$

Let us first consider Compton scattering:

$$
\int d Q\left\langle|\mathcal{M}|^{2}\right\rangle_{c o m p}=\frac{e^{4}}{4 \pi}\left(\ln \frac{s}{\Lambda^{2}}+\frac{1}{2}\right),
$$

where $\Lambda^{2} \ll s$ cuts off the logarithmic infrared divergence of the $u$-channel.

Using $s=2 p k(1-\hat{p} \cdot \hat{k})$ we obtain after integrating over $d^{3} k$

$$
\gamma_{\text {hard }}^{\text {comp }}=\frac{\pi}{12} \frac{\alpha^{2} T^{2}}{p}\left[\ln \frac{8 p T}{\Lambda^{2}}+\frac{1}{2}-\gamma+\frac{\zeta^{\prime}(2)}{\zeta(2)}\right],
$$

where $\gamma=0.57722$ is Euler's constant and $\zeta(z)$ is Riemann's zeta function with $\zeta^{\prime}(2) / \zeta(2)=-0.56996$ [7].

The pair creation contribution gives analogously

$$
\gamma_{\text {hard }}^{\text {pair }}=\frac{\pi}{6} \frac{\alpha^{2} T^{2}}{p}\left[\ln \frac{4 p T}{\Lambda^{2}}-1-\gamma+\frac{\zeta^{\prime}(2)}{\zeta(2)}\right] .
$$

Adding up the (13) and (14) we get the total hard contribution

$$
\gamma_{\text {hard }}=\frac{\pi}{4} \frac{\alpha^{2} T^{2}}{p}\left[\ln \frac{p T}{\Lambda^{2}}+\frac{1}{3} \ln 128-\frac{1}{2}-\gamma+\frac{\zeta^{\prime}(2)}{\zeta(2)}\right] .
$$

Adding up the soft contribution (7) and the hard one (15) the separation scale $\Lambda$ cancels, as expected, yielding the final result

$$
\gamma=\frac{\pi}{4} \frac{\alpha^{2} T^{2}}{p} \ln \frac{0.3090 p}{\alpha T}
$$

Inserting into this expression a typical temperature for a supernova core, $T=10 \mathrm{MeV}$, (neglecting a finite chemical potential) a $100 \mathrm{MeV} \gamma$-ray has a mean free path $\lambda=\gamma^{-1} / 2$ of $0.40 \mathrm{~nm}$.

In the case of a quark-gluon plasma the photon damping is caused by the processes of Fig.1, where the outgoing photon for Compton scattering and one incoming for pair creation has to be replaced by a gluon, leading to

$$
\gamma=\frac{5 \pi}{9} \frac{\alpha \alpha_{s} T^{2}}{p} \ln \frac{0.2317 p}{\alpha_{s} T}
$$

The mean free path of a $1 \mathrm{GeV}$ photon in a QGP at $T=200 \mathrm{MeV}$, extrapolating the result (17) obtained in the weak coupling limit $g \ll 1$ to a realistic $\alpha_{s}=0.3$, is $\lambda=480 \mathrm{fm}$. This value is much larger than the dimensions of a fireball in ultrarelativistic heavy ion collisions confirming that photons may be used as a direct probe of the fireball 
[33,35]. (Of course, higher order processes such as bremsstrahlung may contribute to the rate similarly for realistic values of the strong coupling constant.)

Comparing (17) with the photon production rate $p d R / d^{3} p$ [33, 36] we observe that the latter differs only by a factor $4 /(2 \pi)^{3} \exp (-p / T)$. The Boltzmann factor reflects the principle of detailed balance relating the decay rate to the inverse rate $\Gamma_{i}$ by $\Gamma_{d}(p)=\exp (-p / T) \Gamma_{i}(p)$ [31]. The factor $4 /(2 \pi)^{3}$ is just a matter of definition: $p d R / d^{3} p=$ $2 \Gamma_{i} /(2 \pi)^{3}$ and $\gamma=\Gamma_{d} / 2$. This also shows that the hard part of the photon production rate [33,37] can be calculated much easier by considering the decay instead of the production process.

[1] R.D. Pisarski, Phys. Rev. Lett. 63, 1129 (1989).

[2] V.V. Lebedev and A.V. Smilga, Ann. Phys. (N.Y.) 202, 229 (1990).

[3] V.V. Lebedev and A.V. Smilga, Phys. Lett. B 253, 231 (1991).

[4] V.V. Lebedev and A.V. Smilga, Physica A 181, 187 (1992).

[5] M.H. Thoma and M. Gyulassy, Nucl. Phys. B351, 491 (1991).

[6] E. Braaten, Nucl. Phys. B (Proc. Suppl.) 23B, 351 (1991).

[7] E. Braaten and M.H. Thoma, Phys. Rev. D 44, 1298 (1991).

[8] C.P. Burgess and A.L. Marini, Phys. Rev. D 45, 17 (1992).

[9] A. Rebhan, Phys. Rev. D 46, 482 (1992).

[10] R. Baier, G. Kunstatter, and D. Schiff, Phys. Rev. D 45, 4381 (1992).

[11] H. Nakkagawa, A. Niégawa, and B. Pire, Phys. Lett. B 294, 396 (1992).

[12] A.V. Smilga, Bern preprint No. BUTP-92/39 (unpublished).

[13] T. Altherr, E. Petitgirad, and T. del Rió Gaztelurrutia, Phys. Rev. D 47, 703 (1993).

[14] R. Baier, H. Nakkagawa, and A. Niégawa, Can. J. Phys. 71, 205 (1993).

[15] H. Heiselberg and C.J. Pethick, Phys. Rev. D 47, 769 (1993).

[16] R.D. Pisarski, Phys.Rev D 47. 5589 (1993).

[17] S. Peigné, E. Pilon, and D. Schiff, Z. Phys. C 60, 455 (1993).

[18] M.H. Thoma, Phys. Rev. D 49, 451 (1994).

[19] R. Baier and R. Kobes, Winnipeg preprint, 1994 (unpublished).

[20] E. Shuryak, Phys. Rev. Lett. 68, 3270 (1992).

[21] M.H. Thoma, Phys. Lett. B 269, 144 (1991).

[22] E. Braaten and M.H. Thoma, Phys. Rev. D 44, R2625 (1991).

[23] S. Mrówczyński, Phys. Lett. B 269, 383 (1991).

[24] M.H. Thoma, Phys. Lett. B 273, 128 (1991).

[25] Y. Koike and T. Matsui, Phys. Rev. D 45, 3237 (1992).

[26] A. Rebhan, Phys. Rev D 46, 4779 (1992).

[27] E. Braaten and R.D. Pisarski, Phys. Rev. D 46, 1829 (1992).

[28] R. Kobes and K. Mak, Phys. Rev. D 48, 1868 (1993).

[29] E. Braaten and R.D. Pisarski, Nucl. Phys. B337, 569 (1990).

[30] E. Braaten and T.C. Yuan, Phys. Rev. Lett. 66, 2183 (1991).

[31] H.A. Weldon, Phys. Rev. D 28, 2007 (1983).

[32] E. Braaten, R.D. Pisarski, and T.C. Yuan, Phys. Rev. Lett. 64, 2242 (1990).

[33] J. Kapusta, P. Lichard, and D. Seibert, Phys. Rev. D 44, 2774 (1991).

[34] F. Halzen and A.D. Martin, Quarks and Leptons (John Wiley, New York, 1984).

[35] E.V. Shuryak, Yad. Fiz. 28, 796 (1978) [Sov. J. Nucl. Phys. 28, 408 (1978)].

[36] R. Baier, H. Nakkagawa, A. Niégawa, and K. Redlich, Z. Phys. C 53, 433 (1992).

[37] G. Staadt, W. Greiner, and J. Rafelski, Phys. Rev. D 33, 66 (1986).

FIG. 1. Lowest order Feynman diagrams for photon damping: (a) Compton scattering, (b) pair creation.

FIG. 2. Photon self energy diagrams containing an effective fermion propagator denoted by a blob. 
This figure "fig1-1.png" is available in "png" format from: http://arxiv.org/ps/hep-ph/9405309v1 